\title{
A commentary on Pitfalls of predicting complex traits from SNPS
}

\author{
Gustavo de los Campos and Daniel A. Sorensen
}

In their recent Opinion article (Pitfalls of predicting complex traits from SNPs. Nature Rev. Genet. 14, 507-515 (2013)) $)^{1}$, Wray and co-authors discuss prediction of complex traits using single-nucleotide polymorphisms (SNPs). We would like to further elaborate and qualify some topics.

As stated by Wray and co-authors ${ }^{1}$, knowing the proportion of variance of a trait that is explained by regression on markers in the population $\left(h^{2}{ }_{M}\right)$ is relevant because, in principle, $h^{2}{ }_{M}$ represents the maximum prediction accuracy $\left(R_{T S T}^{2}\right)$ that is achievable in testing (TST) data if marker effects were known ${ }^{2}$. Following one study ${ }^{3}$, Wray and co-authors ${ }^{1}$ suggest estimating $h^{2}{ }_{M}$ using a ratio of variance components that are inferred from a G-BLUP analysis $\left(h_{G-B L U P}^{2}\right)$. However, the realized proportions of allele sharing at markers and at causal loci can be very different ${ }^{4}$ owing to, for example, imperfect marker-causal loci linkage disequilibrium (LD). Consequently, the marker-based model may largely misrepresent the data-generating process; this is exacerbated with unrelated individu$\mathrm{als}^{5}$. Under these conditions, it is not clear that the finite sample estimate of $h^{2}{ }_{G-B L U P}$ is an unbiased estimate of $h^{2}{ }_{M}$ (REF. 5), conseqeuenty, it is not obvious that $R_{T S T}^{2}$ can achieve values equal to the finite sample estimate of $h_{\text {G-BLUP. }}^{2}$. In a recent article ${ }^{5}$, we studied the $R_{T S T}^{2}$ of G-BLUP and its relationship with $h^{2}{ }_{G-B L U P}$. We show analytically that mis-specification of the training-testing (TRN-TST) genomic relationships (owing to, for example, imperfect marker-causal loci LD) can impose a large-sample upper bound on $R^{2}{ }_{T S T}$ that is considerably lower than the finite sample estimate of $h^{2}{ }_{G-B L U P}$. The same study ${ }^{5}$ also presents simulation scenarios with nominally unrelated individuals, where $R_{T S T}^{2}$ can be extremely low in situations with markedly different $h^{2}{ }_{G-B L U P}$, suggesting a tenuous relationship between $h_{G-B L U P}^{2}$ and $R_{T S T}^{2}$, even with moderately large TRN samples.

\section{Assessment of prediction accuracy}

In the models discussed by Wray and coauthors ${ }^{1}, R_{T S T}^{2}$ is expected to be zero when TRN and TST samples are statistically independent ${ }^{5}$. Therefore, we disagree with the statement "problems occur in the validation stage, when data are not fully independent from those in the discovery phase" (REF. 1).

We agree with Wray and co-authors that estimates of $R_{T S T}^{2}$ can be biased if the TST sample is not representative of the population in which predictions will be used. But we cannot reconcile this with the general advice of eliminating individuals in the TST sample based on predetermined thresholds for SNP-based relationships. Each prediction problem has its own level of accuracy, and proper representation may or may not involve realized relationships above such thresholds.

Wray and co-authors ${ }^{1}$ discuss problems due to stratification in TST samples and, as practical advice, suggest including principal component covariates. One study ${ }^{6}$ shows that inclusion of principal components as fixed effects in a G-BLUP analysis ${ }^{3,7}$ leads to a procedure with undesirable statistical properties. The same study ${ }^{6}$ provides statistically sound methods to quantify the relative contribution of each markerderived principal component to estimates of variances and predictions of genetic values. Because principal components are linear functions of genotypes, removing their effects will, by construction, remove genetic signal that is potentially captured by markers. In general, unless the underlying causes of the signals that are captured by a principal-component analysis can be unambiguously interpreted, it is not clear that 'correcting' for their effects will mitigate the problems emerging from having a non-representative TST sample.

Gustavo de los Campos is at the Section on Statistical Genetics, Biostatistics Department, University of Alabama at Birmingham, Birmingham, Alabama 35294, USA.

Daniel A. Sorensen is at the Department of Molecular Biology and Genetics, Faculty of Science and Technology, Aarhus University, PB 50, DK-8830 Tjele, Denmark.

e-mails: gcampos@uab.edu; daniel.alberto.sorensen@gmail.com doi: 10.1038/nrg3457-c1 Published online 18 November 2013

1. Wray, N. R. et al. Pitfalls of predicting complex traits from SNPs. Nature Rev. Genet. 14, 507-515 (2013).

2. Goddard, M. Genomic selection: prediction of accuracy and maximisation of long term response. Genetica 136, 245-257 (2009).

3. Yang, J. et al. Common SNPs explain a large proportion of the heritability for human height. Nature Genet. 42, 565-569 (2010).

4. Hill, W. G. \& Weir, B. S. Variation in actual relationship as a consequence of Mendelian sampling and linkage. Genet. Res. 93, 47-64 (2011).

5. de los Campos, G., Vazquez, A. I., Fernando, R., Klimentidis, Y. C. \& Sorensen, D. Prediction of complex human traits using the genomic best linear unbiased predictor. PLoS Genet. 9, e 1003608 (2013).

6. Janss, L., de los Campos, G., Sheehan, N. \& Sorensen, D. A. Inferences from genomic models in stratified populations. Genetics 192, 693-704 (2012).

7. Yang, J., Lee, S. H., Goddard, M. E. \& Visscher, P. M GCTA: a tool for genome-wide complex trait analysis. Am. J. Hum. Genet. 88, 76-82 (2011).

Acknowledgements

G.d.I.C. acknowledges financial support from the US National Institutes of Health grants R01GM099992 and R01GM101219.

Competing interests statement

The authors declare no competing interests. 